\title{
Potential Benefits of Dynamic Beam Synthesis to Mobile Satellite Communication, Using the Inmarsat 4 Antenna Architecture as a Test Example
}

\author{
R. F. E. Guy \\ Department of Sensor Systems, BAE Systems ATC, West Hanningfield Road, Great Baddow, Chelmsford CM2 8HN, UK \\ Correspondence should be addressed to R. F. E. Guy, ron.guy@baesystems.com \\ Received 22 September 2008; Accepted 17 March 2009 \\ Recommended by Giovanni Toso
}

\begin{abstract}
Present mobile satellite communication systems use large antennas to provide multiple high-gain beams. Each beam covers a fixed geographic cell on the earth. Spatial frequency reuse is provided by synthesising beams with low-power levels over all cells operating at the same frequency. The performance needs for future systems are steadily increasing, leading to higher-gain requirements, which are met by using larger antennas with narrower beams. So the antenna pointing errors become a significant loss factor. An alternative approach is to abandon the use of fixed beams and dynamically synthesise the beams to optimise the antenna performance in real time. This both increases user gain and lowers cofrequency interference whilst also reducing the effects of pointing errors. Simulations, using the Inmarsat 4 antenna architecture as a test example, show that the spatial isolation performance can be significantly improved by using Dynamic Beam Synthesis.
\end{abstract}

Copyright (C) 2009 R. F. E. Guy. This is an open access article distributed under the Creative Commons Attribution License, which permits unrestricted use, distribution, and reproduction in any medium, provided the original work is properly cited.

\section{Introduction}

The fourth generation of Inmarsat satellites provides a good example of an advanced high-capacity, high-gain fixed beam/cell mobile communication system [1]. The L-band Mobile satellite system utilises a $9 \mathrm{~m}$ deployable reflector fed via a 120-element feed array. A digital signal processor (DSP) is used to produce many different beam types that cover the earth and provide different user services. The most demanding of these is the Personal Mobile Communication (PMC) service. This uses $\sim 200$ high-gain narrow spot beams to cover the earth. The beams provide spatial frequency reuse; so cocoloured beams are synthesised to have a high degree of isolation between them. The earth is covered by contiguous cells (nominally hexagonal, as viewed from the satellite) that are fixed with respect to geographic locations on the earth. The beams are changed throughout the day so that they remain aligned to these fixed geographic cells even though the satellite orbit is slightly inclined. Users are assigned to a beam that aligns with the geographic cell they occupy.
However, performance requirements for mobile satellite communication systems are steadily increasing, both in terms of higher antenna gain and frequency reuse capacity. In order to provide the edge of cell edge of coverage (EOC) directivity and the beam roll-off rate, required to achieve interbeam isolation, the beam size will need to decrease. So, many more beam/cells will be required to cover a given geographical area. This will lead to far larger reflectors and feed arrays with many more radiating elements. For conventional fixed beam/cell systems, the antenna pointing error is likely to become a dominant loss factor in the link budget. An alternative is to abandon the fixed beam/cell scheme and form beams that are optimised to individual user locations. This paper uses simulations of a "dynamic beam synthesis" (DBS) process to highlight the potential benefits of this approach.

\section{Fixed Beam System}

The Inmarsat 4 type of antenna system architecture is used as a baseline to compare the fixed beam/cell system to the 
TABLE 1

\begin{tabular}{ll}
\hline Parameter & Value \\
\hline Transmit & $1525-1559 \mathrm{MHz}$ \\
Receive & $1626.5-1660.5 \mathrm{MHZ}$ \\
Polarisation & RHCP \\
Number of antennas & Single Tx/Rx array fed reflector \\
Antenna type & Offset-fed paraboloidal reflector \\
Diameter & 9 metres \\
F/D & 0.53 \\
Feed & 120 helical elements, array diameter $=2.2$ metres \\
Feed position & In front of focal plane \\
Beamformer & Digital Signal Processor, (DSP) with $158 \times 8$ multiport amplifier $\left(\mathrm{MPA}^{*}\right)$ blocks on transmit \\
Beam alignment & Beams aligned with fixed geographic cells \\
Beam reconfiguration & Beams reconfigured throughout the day to compensate for orbit inclination \\
Frequency reuse & Spatial diversity provides frequency reuse between beams \\
\hline
\end{tabular}

* The MPA blocks are used to distribute power more uniformly over the transmit amplifiers and hence increase their efficiency.

alternative of dynamically synthesising the beams making use of the individual user locations. The following outlines the antenna system.

2.1. Frequency Reuse. Spatial frequency reuse allocates adjacent beams different frequencies but reuses the same frequency in more distant beams. To do this efficiently requires the use of a regular hexagonal grid of beams. Figure 1 shows 3, 4, and 7 colour schemes. Each colour represents a different frequency. The colouring principal can be extended to higher-colour schemes such as 9, 12, 13, 16, and 19. An interesting property is that the distance between common colour beam centres is the square root of the number of colours. This relationship also holds for a square lattice.

A typical PMC beamset, which was designed for 7 colour frequency reuse, is shown in Figure 2. This has 228 contiguous, high-gain beams ( $\sim 38 \mathrm{dBi}$ at edge of coverage). To achieve the isolation requirement the peak to edge gain variation of the beams is $\sim 3 \mathrm{~dB}$.

2.2. Future Trends. The trend for next generation satellites will be to increase the gain requirements. A $6 \mathrm{~dB}$ increase implies doubling the reflector diameter and quadrupling the number of feeds. There will also be pressure to increase the frequency reuse factor. Lowering the frequency colour scheme from 7 to 4 could require the peak to edge gain delta to increase by a further 1 to $2 \mathrm{~dB}$. The exact figure is dependent on the isolation requirement.

2.3. Limitations of a Fixed Beam System. A fixed beam system is limited by the following factors.

(i) Cells fixed at specific geographic location.

(ii) Pointing errors are accounted for by expanding the geographic cells by the pointing error. This degrades both EOC gain and isolation.

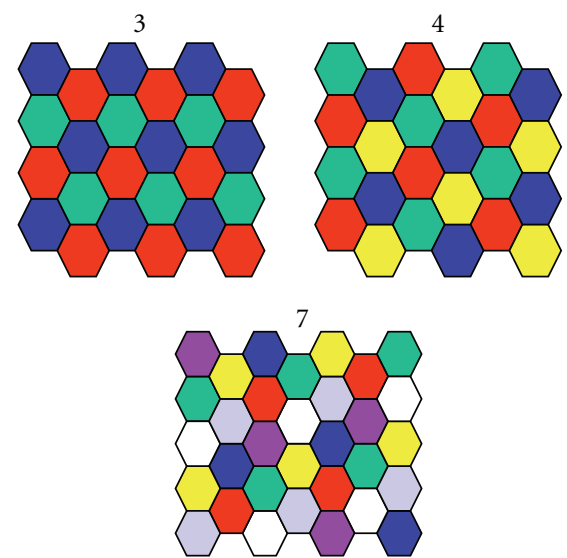

Figure 1: Frequency reuse schemes with 3, 4, and 7 colours.

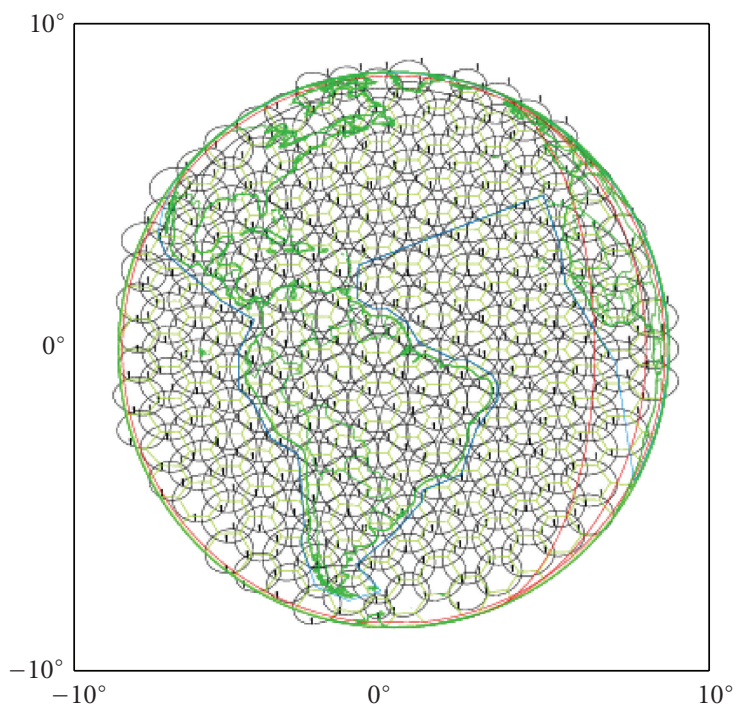

Figure 2: Example fixed beamset. 
(iii) Orbit inclination distorts the fixed cell grid, requiring resynthesis of the whole beamset and the use of new weights many times a day.

(iv) The G/T figure of merit for the satellite is limited by what can be achieved at the worst point at the edge of cell grid, when distorted by the inclination and expanded by the pointing error. This is further reduced by other effects such as vector weight errors.

(v) The EIRP figure of merit for the satellite is limited by the same effects.

2.4. Consequences of Pointing Errors. The following points highlight the effect of pointing error in a fixed beam system.

(i) For the example beamset using a 7-colour reuse scheme, $0.1^{\circ}$ pointing error gives $\sim 0.6 \mathrm{~dB}$ loss of gain at EOC.

(ii) For a scaled system with $6 \mathrm{~dB}$ more gain, $0.1^{\circ}$ pointing error gives $\sim 1.2 \mathrm{~dB}$ loss.

(iii) For a scaled system with $6 \mathrm{~dB}$ more gain, using a 4colour scheme, $0.1^{\circ}$ pointing error gives $\sim 2 \mathrm{~dB}$ loss.

Assuming the pointing error is not significantly reduced for the larger reflector diameters, then as the gain and frequency reuse factor is increased, still further, the pointing error will start to dominate the loss budget.

2.5. Implications of Using a Fixed Beam System. If a fixed beam system is used to meet future gain and isolation demands, the cell size must reduce, and both the reflector diameter and number of feed elements must increase. A $6 \mathrm{~dB}$ gain improvement requires doubling the reflector diameter and quadrupling the number of feeds.

If the reuse scheme falls from 7 to 4 colour, then the additional losses increase by $\sim 3 \mathrm{~dB}$ requiring a $\sim 9 \mathrm{~dB}$ improvement, which implies about three times the diameter reflector and eight times as many elements.

\section{Dynamic Beam System}

In a dynamic beam system the beams are not fixed. The beams are synthesised in "real time," using the known user locations to both maximise individual user gain and minimise interference from other cofrequency users.

3.1. The Synthesis Process. The following outlines the dynamic beam synthesis process.

(1) A fixed geographic cell lattice is used to assign frequencies to users, for example, using 7-colour reuse.

(2) At any one time there are likely to be a number of users assigned the same frequency.

(3) In any one cell only one user has the same frequency.

(4) A new user requests a channel via a global beam, giving their location, (e.g., provided by a GPS receiver). Their geographic location is used to assign an available channel frequency for that user cell.

(5) All other users with the same frequency are identified.

(6) The beams associated with every one of these users are resynthesised every time a new user is assigned an existing frequency.

(7) Each beam is resynthesised to maximise power at the user location and minimise power at the other user locations.

(8) The synthesis expands the user locations by the pointing error to provide coverage and isolation in the presence of pointing errors.

(9) The pointing error allowance would be further expanded to provide extra margin to cope with vector weight errors, and satellite inclination change.

(10) The weights would be resynthesised and updated on a periodic basis to cope with gross inclination changes.

3.2. Simulation of the DBS Process. The simulation program, MAXIM, has been written to simulate the dynamic beam synthesis process. It performs both the synthesis and the analysis to produce plots of directivity and isolation performance. The program works in the following order.

(1) Reads secondary array fed reflector (AFR) element patterns.

(2) Reads cell lattice coordinates and reuse colour scheme.

(3) Chooses a far-field grid point.

(4) Finds associated cell colour and finds all cocoloured cell coordinates.

(5) Randomly assigns " $N$ " other users in "N" different cocoloured cells at random points in each cell.

(6) Synthesises ideal weights for each cocoloured user, using the inverse of the covariance matrix [2].

(7) Calculates beams from the synthesised weights (this step can include vector weight errors if requested).

(8) Calculates the directivity of the user with pointing errors.

(9) Calculates the isolation of the user with pointing errors.

(10) Repeats simulation " $M$ " times and selects requested percentage confidence level performances.

(11) Repeats simulation steps (3)-(10) for all grid points.

(12) Outputs results.

3.3. Simulation Results. The DBS beams were synthesised to maximise power over the user and minimise power over cocoloured users. To illustrate this, the following example is used. A user is at $\left(0.08^{\circ}, 0.08^{\circ}\right)$, and seven other cocoloured user locations are chosen at random. The closest user is in the first cocoloured ring of cells surrounding the coverage 


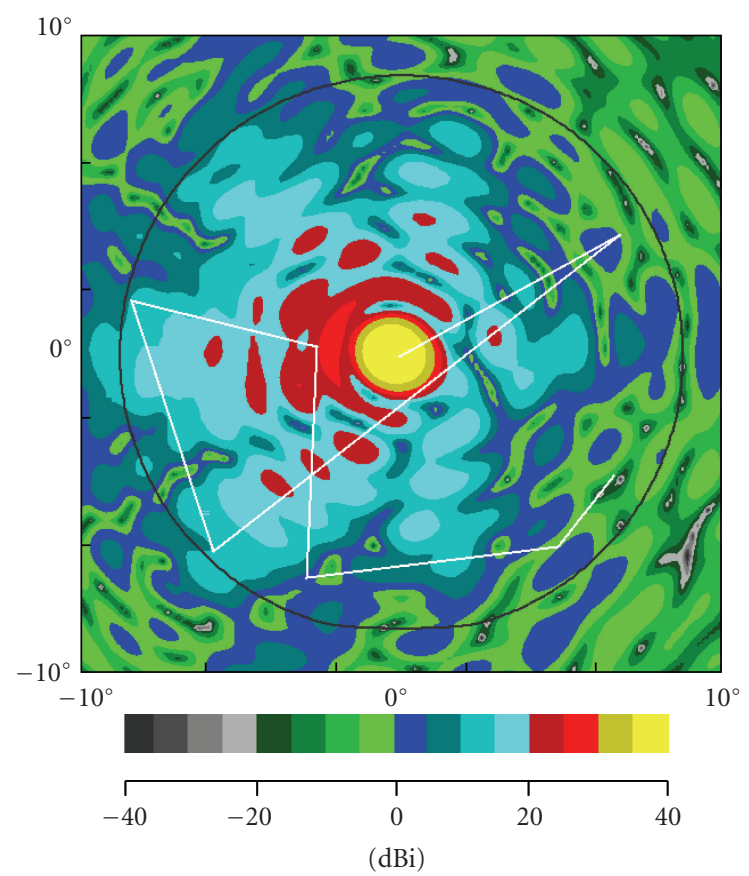

FIgURE 3: Beam prior to DBS.

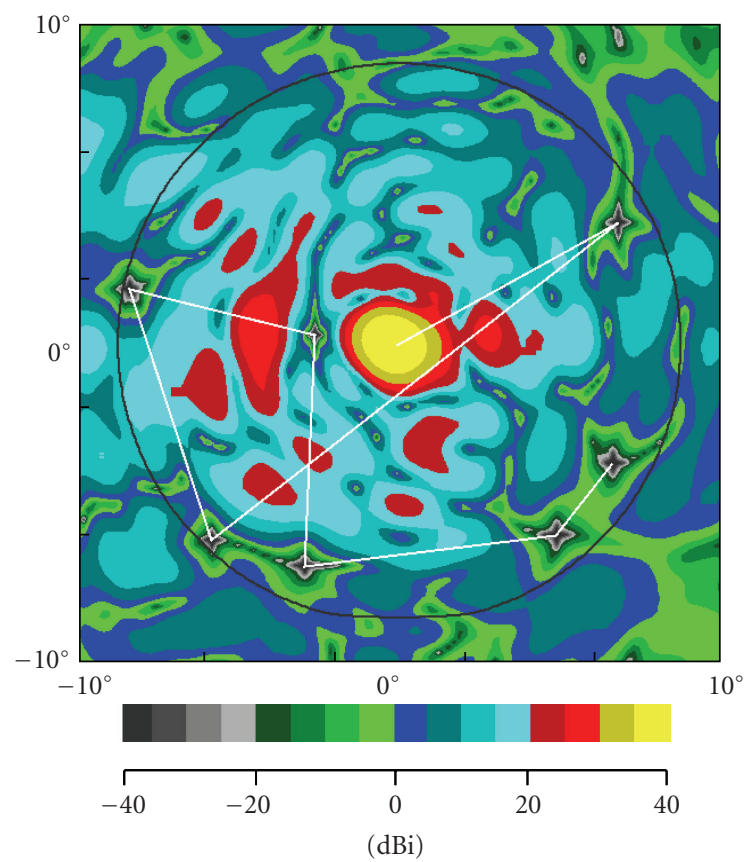

FIgURE 4: Synthesised beams with nulls, no errors.

cell. Figure 3 shows a maximum directivity beam for $\left(0.08^{\circ}\right.$, $0.08^{\circ}$ ), and the locations of the other users are indicated by the vertices of the straight white lines. The beam peak is $43.5 \mathrm{dBi}$, and the level at the closest user is $25 \mathrm{dBi}$ (i.e., $18.5 \mathrm{~dB}$ below the beam peak).

Figure 4 shows the synthesised beam with deep nulls over the other user locations. The nulls are below $-50 \mathrm{dBi}$. The peak directivity has fallen by about $0.25 \mathrm{~dB}$. Figure 5 shows

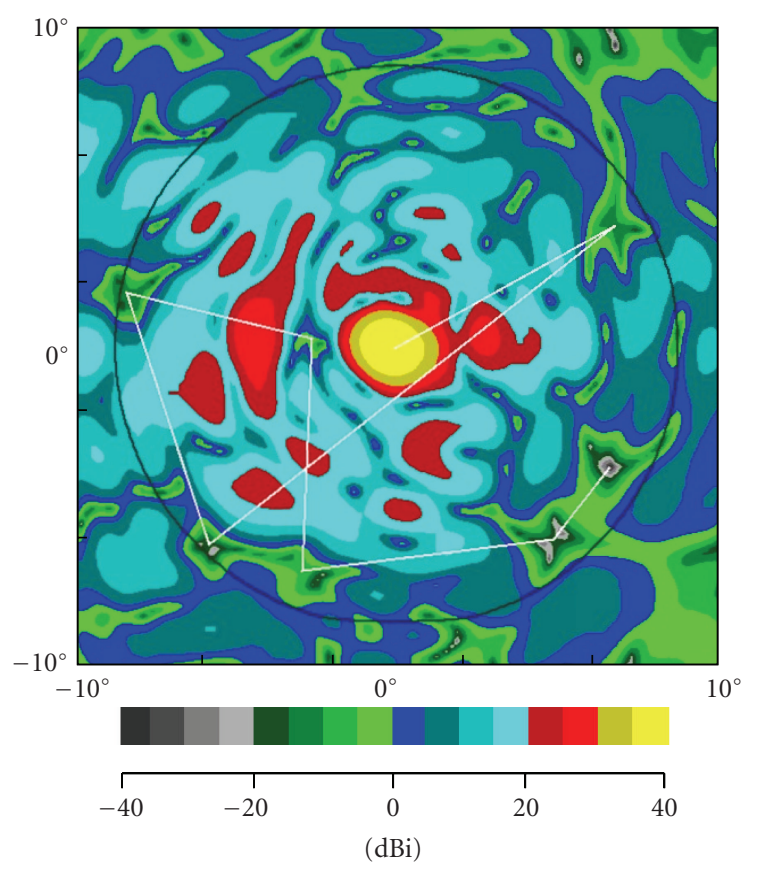

Figure 5: Synthesised beams with nulls, with errors.

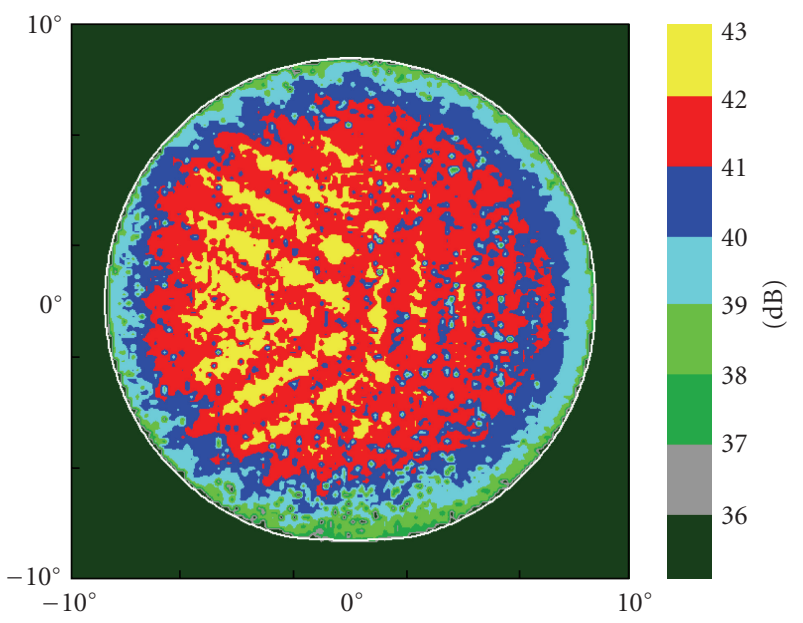

FIgURE 6: Minimum directivity of DBS beams biased for isolation, 7 colour, with errors, (scale 35 to $43 \mathrm{dBi}$ ).

the effect of likely vector weight errors. The nulls have filled in; for example, the level of the closest user is $-1 \mathrm{dBi}$ (i.e., $26 \mathrm{~dB}$ lower than the maximum directivity case). All nulls remain below $0 \mathrm{dBi}$ over an area encompassing the pointing error uncertainty margin and provide $\sim 40 \mathrm{~dB}$ isolation.

A full simulation was carried out over the earth using five trials at each grid point. The worst values, for each grid point, of these five trials were selected. It included vector weight errors, as for the "fixed beamset", and pointing errors of $0.16^{\circ}$. Satellite pointing error margin was $0.1^{\circ}$; the additional $0.06^{\circ}$ is equivalent to a user location error of $40 \mathrm{Km}$ at the subsatellite point. GPS can give user location to tens of meters or better. The directivity levels are shown in Figure 6 . The received isolation over the earth is shown in Figure 7. 


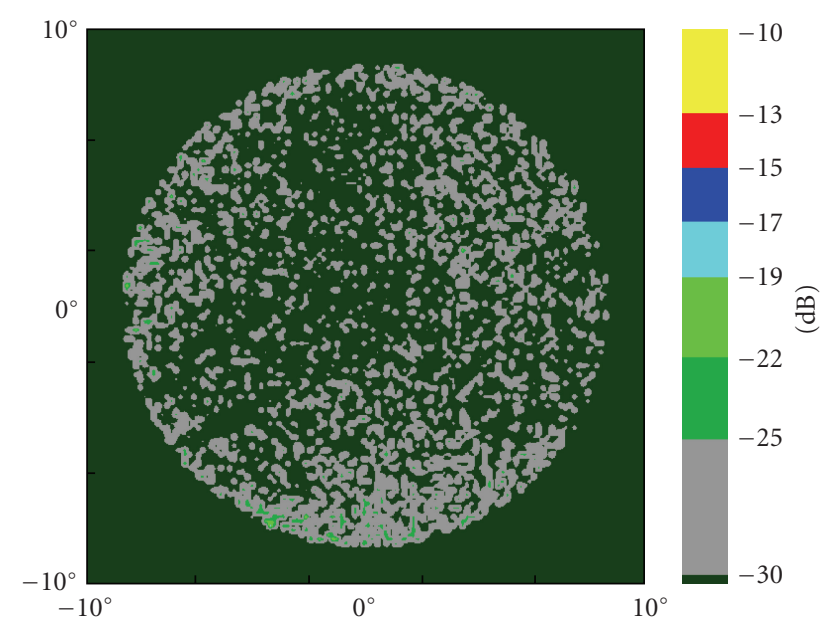

FIGURE 7: Minimum isolation of DBS beams biased for isolation, 7 colour, with errors, (scale -31 to $-10 \mathrm{~dB})$.

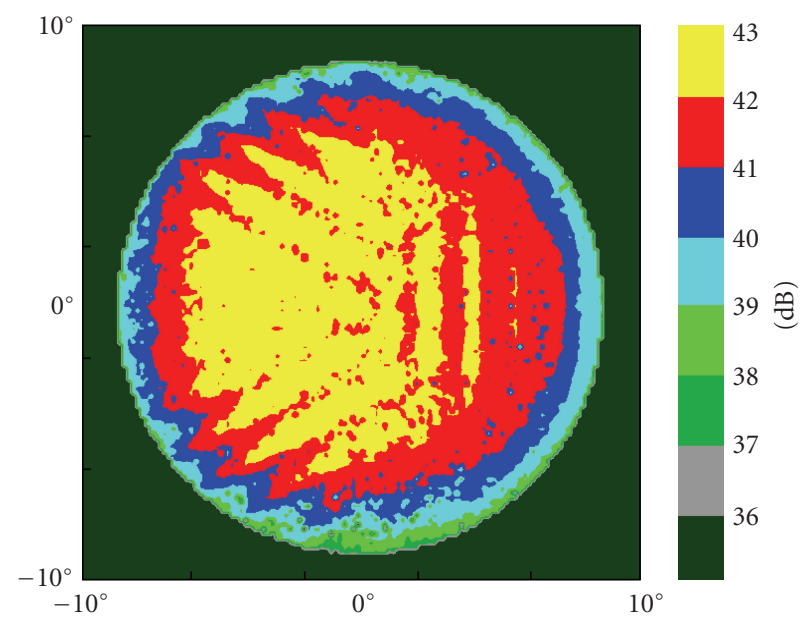

FIgURE 8: Minimum directivity of DBS beams, biased for gain, 7 colour, with errors, (scale 35 to $43 \mathrm{dBi}$ ).

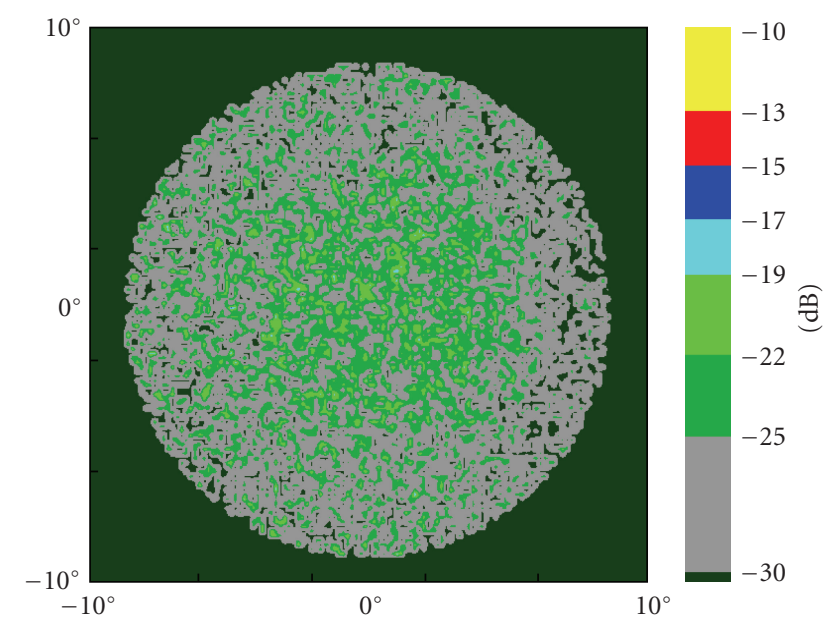

Figure 9: Minimum isolation of DBS beams, biased for gain, 7 colour, with errors, (scale -31 to $-10 \mathrm{~dB})$.
The received isolation was calculated as the ratio of the sum of the powers received from the cocoloured users to the power of the user. The results presented in this paper are for a 7-colour frequency reuse scheme, with eight cocoloured users.

The results show higher directivity levels than the fixed beamset. There are a few areas where the directivity was lower than before. This was due to the synthesis procedure achieving very low nulls at the expense of user directivity. Higher directivity levels can be achieved by relaxing the null depth. Figures 8 and 9 show the directivity and isolation when the synthesis is biased more towards directivity.

\section{Conclusion}

The "dynamic beam synthesis" (DBS) process has the potential to increase both the gain and isolation performance of multibeam, frequency reuse, and satellite communication systems. The simulations show that the isolation performance of dynamic beam synthesis can be an order of magnitude better than using the fixed beam case.

\section{References}

[1] R. F. E. Guy, C. B. Wyllie, and J. R. Brain, "Synthesis of the Inmarsat 4 multibeam mobile antenna," in Proceedings of the 12th International Conference on Antennas and Propagation (ICAP '03), vol. 1, pp. 90-93, Exeter, UK, March-April 2003.

[2] R. T. Compton, Adaptive Antennas, Prentice-Hall, Englewood Cliffs, NJ, USA, 1988. 

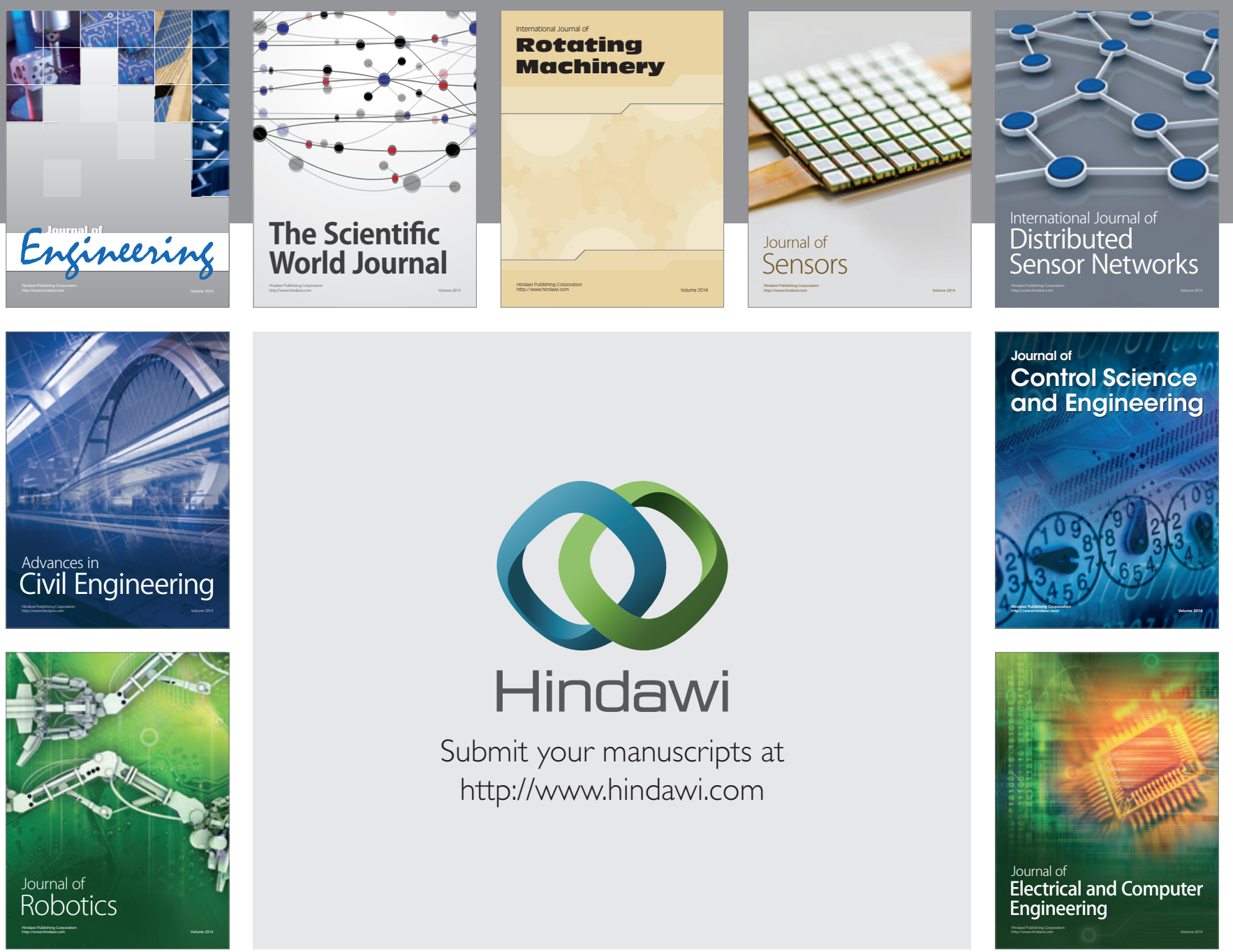

Submit your manuscripts at

http://www.hindawi.com
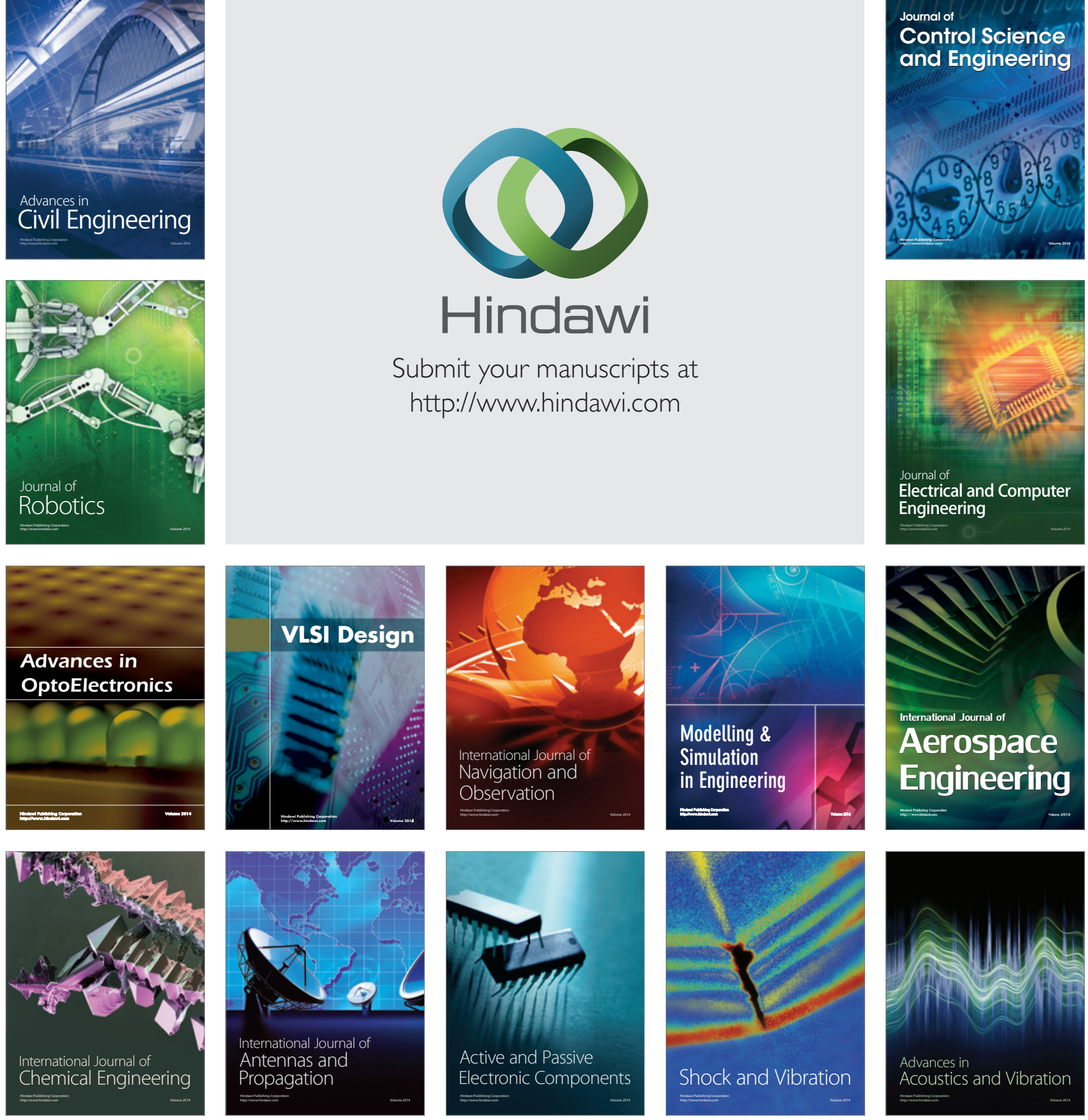